\title{
Current Views on the Structural Study of Interface of Liquids and Surface Layer of Solids
}

\author{
Yoshio WASEDA and Eiichiro MATSUBARA
}

Research Institute of Mineral Dressing and Metallurgy, Tohoku University, Katahira, Aoba-ku, Sendai, Miyagi-ken, 980 Japan.

(Received on June 4, 1991; accepted in final form on September 20,1991)

\begin{abstract}
Various phenomena of metallurgical interest are well-known to occur through the liquid-vapor and/or liquid-solid interfaces. Thus, a knowledge of the structure of interface of liquids and surface layers of solids is essential for profound understanding of these subjects. An attempt will be made in this paper to review some current information on the structure of liquid-vapor and solid-liquid interfaces, and a surface layer of solid determined from $X$-ray reflectivity measurements, grazing incidence $X$-ray scattering, and conventional and anomalous $X$-ray scattering measurements for fine particles with a crystalline size of about $10 \mathrm{~nm}$ or less. Some theoretical studies relating with these interfaces are also included for further convenience.
\end{abstract}

KEY WORDS: interface; interfacial energy; surface tension; fine particles; transition zone; surface layer; grazing incidence $X$-ray scattering; $X$-ray reflectivity; radial distribution function.

\section{Introduction}

Various reactions through the liquid-vapor and liquid-solid interfaces are known to play a significant role in many metallurgical processes. There is, therefore, an increasing need for the information of the structures of these interfaces. A number of the experimental data are available, for example surface tension of liquid metals and slags using the maximum bubble method or solidliquid interfacial energy of metals determined from the dihedral angle measurements. However, the structural studies of interface of liquids and surface layer of solids are still limited and the knowledge of interfaces at the atomic level is far from complete. Of course, from the basic scientific point of view, thermodynamics and atomic structures of liquid-vapor, liquid-solid, and solid-vapor interfaces are subjects of intensive experimental and theoretical interest. The classical picture of these interfaces as a zero thickness junction between two homogeneous semi-infinite phases is inconsistent with some recent experimental results obtained by several experimental techniques which are highly sensitive to atomic structures in the interface. Among these new techniques, structural studies of liquid-vapor and liquid-solid interfaces using X-ray diffraction will be described in this review as well as theoretical studies relating with these interfaces.

\section{Liquid-Vapor Interface}

\subsection{Theoretical Studies}

A general statistical mechanical theory of interfacial phenomena of simple liquids developed by Kirkwood and $\mathrm{Buff}^{1)}$ provides a rigorous expression for the surface tension. A similar discussion has also been given by Harashima $^{2)}$ using the partition function. According to Kirkwood and Buff, ${ }^{11}$ the surface tension is estimated from the interatomic potential, the pair distribution function and the number density. Their approach, however, is limited by the lack of the pair distribution function on the liquid-vapor interface, i.e. the liquidvapor transition zone. On the other hand, pair distribution functions of bulk liquids in various systems have already been determined with sufficient accuracy, using $\mathrm{X}$-ray and neutron diffraction. In order to obtain a numerical example, an appropriate assumption of the atomic distribution in the liquid-vapor transition zone is required. The simplest approximation is that liquid properties change abruptly from their liquid to their vapor values. When the vapor density is equal to zero (namely, infinitely dilute) in this approximation, the Kirkwood-Buff formula is reduced to an earlier expression by Fowler ${ }^{3)}$ in a less general way. The resultant simplified expression for the surface tension $\gamma$ of liquid becomes

$$
\gamma=\frac{1}{8} \pi \rho_{0}^{2} \int_{0}^{\infty} g(r) \frac{\mathrm{d} \Phi(r)}{\mathrm{d} r} r^{4} \mathrm{~d} r
$$

where $\phi(r)$ is the interatomic potential, $g(r)$ the pair distribution function and $\rho_{0}$ the average number density.

Shoemaker et $\mathrm{al}^{.4)}$ systematically calculated the surface tension of various liquids using Eq. (1), the pair distribution function available from diffraction experiments, and Lennard-Jones potential function constants from the pressure and internal energy integrals. These 
Table 1. Properties of liquid-vapor transition zone of simple liquids. ${ }^{4,5}$

\begin{tabular}{lcrrlll}
\hline \multicolumn{1}{c}{$\begin{array}{c}\text { Tempera- } \\
\text { ture } \\
(\mathrm{K})\end{array}$} & $\begin{array}{c}\gamma\left(10^{-3} \mathrm{~N} / \mathrm{m}\right) \\
\text { exp. }\end{array}$ & cal. & $L(\mathrm{~nm})$ & $r_{\mathrm{o}}(\mathrm{nm})$ & $3 L / r_{\mathrm{o}}$ \\
\hline Argon & 84.3 & 13.2 & 15.1 & 0.11 & 0.36 & 0.92 \\
Krypton & 117 & 16.1 & 17.1 & $0.13^{*}$ & $0.36^{*}$ & $1.08^{*}$ \\
Xenon & 161.5 & 19.3 & 24.5 & $0.15^{*}$ & $0.39^{*}$ & $1.15^{*}$ \\
Neon & 33.1 & 2.7 & 4.5 & 0.31 & 0.31 & 3.0 \\
Nitrogen & 64 & 12.0 & 12.7 & 0.17 & 0.38 & 1.36 \\
& 77 & 8.9 & 11.5 & 0.265 & 0.39 & 2.03 \\
Oxygen & 77 & 16.5 & 17.6 & 0.156 & 0.35 & 1.34 \\
Methane & 96 & 16.0 & 15.8 & 0.12 & 0.39 & 0.92 \\
& 121 & 11.3 & 14.6 & 0.25 & 0.40 & 1.83 \\
\hline
\end{tabular}

* Y. Waseda

values are listed in Table 1 together with the experimental data. The calculated values are about $25 \%$ larger than the experimental data for all cases except for liquid neon. This discrepancy, however, is acceptable considering the extremely simplified model for the liquid-vapor transition zone.

Berry et al. $^{5)}$ proposed a practical method for calculating the surface tension of liquids, assuming a smooth variation of density between liquid and vapor (in contrast to the abrupt change of the density in the Fowler theory). When a density is given by $\rho(z)$ where $z$ is a coordinate perpendicular to the interface, $z \gg 0$ being in the vapor, $z \ll 0$ in the liquid, and $z=0$ somewhere in the interface, by introducing the following simple exponential form for the density in the liquid-vapor transition zone;

$$
\begin{array}{ll}
\rho(z)=\rho_{\mathrm{o}}\left[1-\frac{1}{2} \exp (z / L)\right] & z<0 \quad \text { (liquid side) } \\
\rho(z)=\frac{1}{2} \rho_{\mathrm{o}} \exp (-z / L) & z>0 \quad \text { (vapor side) }
\end{array}
$$

the rigorous formula for surface tension given by Kirkwood and Buff ${ }^{1)}$ is rewritten in the following simple form containing the single adjustable parameter $L$,

$$
\begin{aligned}
& \gamma=\frac{\pi}{8} \rho_{0}^{2} \int_{0}^{\infty} g(r) \frac{\mathrm{d} \Phi(r)}{\mathrm{d} r} f(L) \mathrm{d} r \\
& f(L)=L^{4}\left[\xi^{2}\left(\xi^{2}-8\right)+72-e^{-\xi}\left\{4 \xi\left(\xi^{2}+7 \xi+18\right)+72\right\}\right]
\end{aligned}
$$

where $\xi=r / L$, and Eq. (3) is readily reduced to Eq. (1) for $L=0$.

Berry et al. ${ }^{5)}$ estimated the surface tension of simple liquids as a function of $L$ using Eq. (3) and the same constants for interatomic potentials of Shoemaker et al. ${ }^{4)}$ Their calculated results are shown in Fig. 1, where solid and open circles denote the experimental data and the values calculated by Eq. (1), respectively. The curves in Fig. 1 imply that the calculated values using Eq. (3) are reduced with increase in the parameter of $L$.

Here, let us take $3 L$ as the thickness of the liquid-vapor transition zone, and a quantity of $\left(3 L / r_{0}\right)$ is obtained for the number of atomic layers, where the value of $r_{\mathrm{o}}$ is the interatomic distance estimated by $r_{\mathrm{o}}=\{$ (atomic weight $) /$

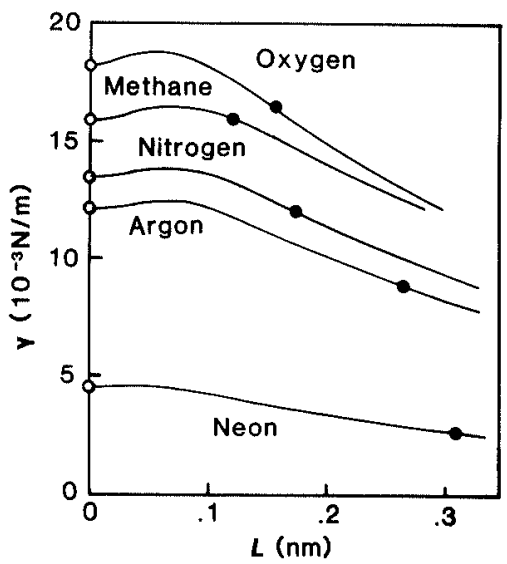

Fig. 1. Calculated surface tension $(\gamma)$ as a function of the interfacial thickness parameter $(L) .{ }^{5)}$ Solid and open circles denote the experimental data and the values calculated by Eq. (1)

Table 2. Properties of liquid-vapor transition zone in liquid metals. ${ }^{9)}$

\begin{tabular}{lccccc}
\hline \multicolumn{5}{c}{$\begin{array}{c}\text { Tempera- } \\
\text { ture } \\
(\mathrm{K})\end{array}$} & $\begin{array}{c}\gamma \\
\end{array}$ \\
& $\left(10^{-3} \mathrm{~N} / \mathrm{m}\right)$ & $L(\mathrm{~nm})$ & $r_{\mathrm{o}}(\mathrm{nm})$ & $3 L / r_{\mathrm{o}}$ \\
\hline $\mathrm{Na}$ & 373 & 191 & 0.34 & 0.333 & 3.1 \\
$\mathrm{~K}$ & 338 & 101 & 0.38 & 0.444 & 2.8 \\
$\mathrm{Rb}$ & 313 & 78 & 0.39 & 0.433 & 2.7 \\
$\mathrm{Al}$ & 943 & 914 & 0.26 & 0.246 & 3.2 \\
$\mathrm{~Pb}$ & 613 & 480 & 0.28 & 0.291 & 2.9 \\
\hline
\end{tabular}

(density) $\left./ N_{\mathrm{o}}\right\}^{1 / 3}$ ( $N_{\mathrm{o}}$ being Avogadro's number). The resultant values ${ }^{5)}$ are given in Table 1 . From these results, it is concluded that the thickness of the liquid-vapor transition zone in simple liquids is the order of one or two atomic layers. These values show a surprisingly fair agreement with the values estimated by other methods. ${ }^{6-8)}$

A similar method for estimating the surface tensions of several liquids has been done ${ }^{9)}$ and the results are given in Table 2. A few atomic layers are obtained for the liquid-vapor transition zone in liquid metals, which is consistent with the conclusion drawn from the analyses of isothermal compressibility and surface tension for various liquid metals. ${ }^{10)}$ Small difference between simple liquids and liquid metals may be explained by the difference of the interatomic potentials. Namely, liquid metals have the long-range oscillatory type and simple liquids have the short-range Lennard-Jones type. It may also be worth mentioning that the experimental measurements of the surface tension for liquids presently investigated are subject to uncertainties of at most $5 \%$ for all cases. ${ }^{1,12)}$ Thus, these experimental errors suggest an uncertainty of about $0.04 \mathrm{~nm}$ for the parameter $L$.

Further theoretical studies and direct measurements for the liquid-vapor transition zone are required by all means. The present authors maintain the view from the information mentioned above that a diffuse surface layer is not suggested in liquids and the density gradient at the liquid-vapor interface is relatively sharp with a few atomic layers. 


\subsection{Experimental Studies}

In our present understanding, an atomic structure of the liquid-vapor interface has been deduced from both macroscopic measurements and computer simulations. Data from macroscopic measurements, such as the surface tension, are not extremely sensitive to the structure of the interface. On the other hand, computer simulations effectively visualize the structure of the interface. However, their computations involve some physical assumptions. Consequently, for further development of structural studies of the interface, these theoretical results must be compared with the structural data experimentally determined.

Atomic structures of the bulk phases of liquid metals and simple liquids are accurately determined by the usual $\mathrm{X}$-ray and neutron diffraction techniques. In the usual experimental arrangements, X-rays and neutrons, however, deeply penetrate into liquids, hence determining the atomic structure of a liquid-vapor interface is compromised by an extremely small ratio of the number of atoms in the interface to that in the bulk. This difficulty of the diffraction experiment with $\mathrm{X}$-rays for surface is solved by using the X-ray reflection geometry.

Because the reflective index of $X$-rays for most substances is slightly less than one, the total external reflection of X-rays is observed from a smooth surface

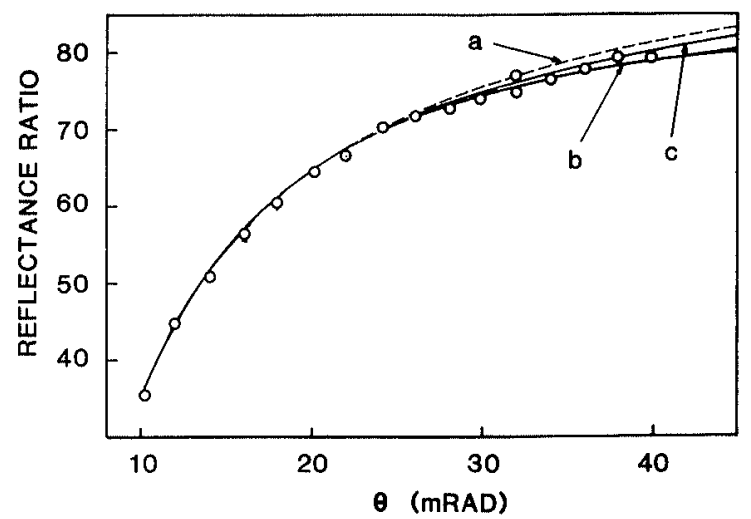

Fig. 2. Comparison of experimental data ${ }^{14)}$ (open circles) with the predicted X-ray reflectivity from (a) a zero thickness transition zone (upper dashed curve), (b) the best fitted monotone density profile, ${ }^{14)}$ and (c) D'Evelyn and Rice profile. ${ }^{16)}$

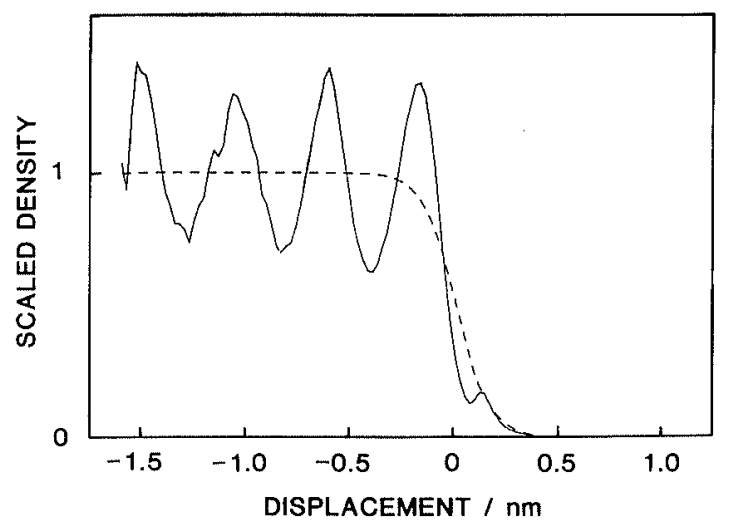

Fig. 3. D'Evelyn and Rice (solid curve) ${ }^{16)}$ and the best fitted monotone (dashed curve) ${ }^{15)}$ density profiles at liquid-vapor interface. at a small glancing angle of incidence, which was originally demonstrated by Compton. ${ }^{13)} \mathrm{X}$-rays are strongly attenuated in the total reflection condition and their penetration is limited to the order of a few $\mathrm{nm}$. Parratt $^{14)}$ proposed a method for studying certain structural properties of the surface about several to $10 \mathrm{~nm}$ deep by analyzing the profiles of the X-ray reflection intensity. Parratt ${ }^{14)}$ obtained the reflection curves from $\mathrm{Cu}$ and oxidized $\mathrm{Cu}$ surfaces, and developed models for the spatial dependence of surface oxidation.

Sluis and Rice ${ }^{15)}$ applied this method, measured the angular dependence of the reflection from liquid-vapor interface of $\mathrm{Cs}$ and compared the experimental profile with the theoretical ones estimated from several models for the interface.

In Fig. 2, the experimentally observed ratio of the $X$-ray reflectivity at angle $\theta$ to that at angle $(\theta-5) \mathrm{mrad}$ is compared with the predicted $X$-ray reflectivities from Fowler's interface of a zero thickness transition zone, the density profile by D'Evelyn and Rice, ${ }^{16)}$ and the best fitted monotone density profile. ${ }^{15)}$ D'Evelyn and Rice's profile $^{16)}$ predicted from Monte Carlo calculations consists of stable density oscillations, with spacing about one atomic diameter, extending several layers into the bulk liquid. The actual density profile is shown in Fig. 3 with the best fitted monotone density profile. In Fig. 2 , the best fitted monotone density profile gives almost as good a fit to the experimental data as does the D'Evelyn-Rice profile. Thus, although D'Evelyn and Rice's model is one of the most plausible choice for the Cs liquid-vapor interface structure as Sluis and Rice mentioned in their paper, ${ }^{15)}$ the $\mathrm{X}$-ray reflectivity data are not sufficient enough to give the definite comments on the superiority of any proposed structures of the interface in the present stage. In order to overcome such a problem of the reflectivity measurements, Mohanty and Rice $^{17)}$ emphasized the use of the total external X-ray reflectance-diffraction together with the X-ray reflection. They derived a formula for the structure factor of the liquid-vapor transition zone in this new reflectancediffraction geometry. The details of the total external $\mathrm{X}$-ray reflectance-diffraction method will be given in the following section.

\section{Liquid-Solid Interface}

\subsection{Theoretical Studies}

Liquid-solid interfacial energy of materials is one of the important physical parameters in controlling a wide range of phenomena, such as the rate of sintering and the growth rate during particle coarsening. Solidification theories for interpreting crystal nucleation and growth almost inevitably include the liquid-solid interfacial energy. For this reason, there have been many attempts, both experimental and theoretical, to estimate the values of the interfacial energy for various materials. ${ }^{18,19)}$ However, the experimental determination of the absolute values of the liquid-solid interfacial energy is not so easy task for any type of materials. Thus, by far the most widely used experimental values of the liquid-solid interfacial energy of metals are those estimated from the 
homogeneous nucleation studies by Turnbull and Cech. $^{20)}$ On the other hand, theoretical studies for estimating the liquid-solid interfacial energy are extremely complicate although there is some hope that the recent progress will provide reliable calculations for metals. Thus, we will here present only some works in simple metals for simplification.

An earlier model of such as Jackson and Chalmers, ${ }^{21)}$ Skapski, ${ }^{21}$ Zadumkin, ${ }^{23)}$ and Kotze and KuhlmannWilsdorf, ${ }^{24)}$ etc. includes only the solid phase contribution to the interfacial energy. In spite of the minor difference, all these models include the assumptions that the entropy of both liquid and solid phases and the energy density of the liquid are constant up to the interface and that the energy density of the solid increases in a step-wise fashion at the interface due to missing nearest neighbors. Turnbull ${ }^{25}$ pointed out that the liquid phase also should contribute to the liquid-solid interfacial energy, owing to a reduced liquid entropy in close vicinity to the liquid-solid interface where atomic positions in the liquid phase are severely restricted. Ewing ${ }^{26)}$ proposed a new model for the liquid-solid interfacial energy of metals considering Turnbull's suggestion.

Ewing ${ }^{26,27)}$ proposed that the liquid-solid interfacial energy $\gamma_{C L}$ can be given by the two terms

$$
\gamma_{\mathrm{CL}}=\gamma_{\mathrm{CL}}^{\mathrm{S}}+\gamma_{\mathrm{CL}}^{\mathrm{L}}
$$

where $\gamma_{\mathrm{CL}}^{\mathrm{S}}$ is the contribution of the solid to the interfacial energy associated with the solid, and $\gamma_{\mathrm{CL}}^{\mathbf{L}}$ is the contribution of the liquid near the interface, which is caused by the structural constraints to the liquid phase by the adjacent crystalline solid phase. The $\gamma_{\mathrm{CL}}^{\mathrm{s}}$ term is estimated in the close packed crystalline plane by assuming that one-quarter of a surface atom's bonding is liquid rather than crystal bonding, and that the contribution of the crystal surface to the interfacial free energy is onequarter of the latent heat of fusion $\Delta H_{\mathrm{f}}{ }^{22}{ }^{2}$ )

$$
\gamma_{\mathrm{CL}}^{\mathrm{S}}=n_{\mathrm{S}} \Delta H_{\mathrm{f}} / 4 N_{\mathrm{o}}
$$

where $n_{\mathrm{S}}$ is the number density in the surface plane, $\Delta H_{\mathrm{f}}$ the latent heat of fusion per mole and $N_{\mathrm{o}}$ is Avogadro's number.

In order to estimate the term $\gamma_{\mathrm{CL}}^{\mathrm{L}}$, Ewing ${ }^{26)}$ assumed a physically plausible structure of the interface. The atomic ordering in the bulk solid remains up to the dividing interface and the atomic distribution in the liquid along the surface normal at the interface is identical to the so-called radial distribution function of the bulk liquid. The profile of typical atomic arrangements at the close vicinity of a liquid-solid interface along the surface normal is schematically shown in Fig. 4. Since the poisition of atoms in the liquid near the liquid-solid interface is severely restricted by the solid surface, the liquid entropy near the solid surface $\Delta S_{\mathrm{CL}}$ is reduced. ${ }^{25)}$ Using the above approximation, the value $\Delta S_{\mathrm{CL}}$ is given as follows:

$$
\Delta S_{\mathrm{CL}}=n_{\mathrm{L}} k_{\mathrm{B}} \int_{0}^{b} g(r) \ln g(r) \mathrm{d} r
$$

where $n_{\mathbf{L}}$ is the number density of liquid atoms in the

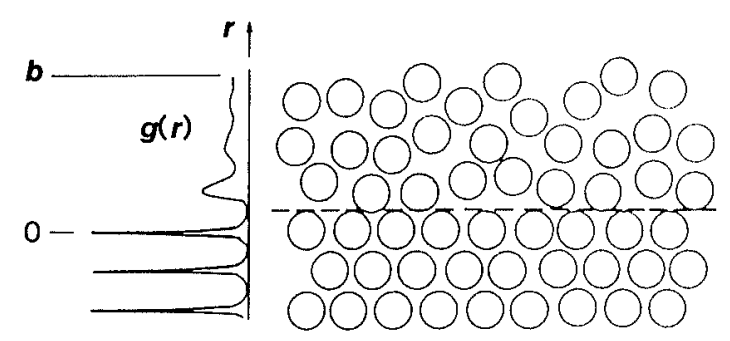

Fig. 4. A typical arrangement of atoms at the interface and the corresponding normal distribution function. ${ }^{26)}$

Table 3. Liquid-solid interfacial free energy ${ }^{29)}$ in unit of $\mathrm{mJ} / \mathrm{m}^{2}$ from experimental radial distribution function data estimated by the method of Ewing. ${ }^{26)}$

\begin{tabular}{|c|c|c|c|c|c|}
\hline Metal & $\begin{array}{c}\text { Crystal } \\
\text { structure } \\
\text { at } T_{m}\end{array}$ & $\gamma_{\mathrm{CL}}^{\mathrm{s}}$ & $\gamma_{\mathrm{CL}}^{\mathrm{L}}$ & $\begin{array}{c}\gamma_{\mathrm{CL}} \\
\text { (calc.) }\end{array}$ & $\begin{array}{c}1 / \mathrm{CL} \\
\text { (exp.) }\end{array}$ \\
\hline $\mathrm{Ag}$ & $\mathrm{FCC}$ & 76.0 & 80.9 & 156.9 & 140.9 \\
\hline $\mathrm{Al}$ & FCC & 74.5 & 66.5 & 141.0 & 102.0 \\
\hline $\mathrm{Au}$ & $\mathrm{FCC}$ & 85.5 & 82.2 & 167.7 & 146.1 \\
\hline $\mathrm{Ba}$ & $\mathrm{BCC}$ & 23.3 & 13.4 & 36.7 & \\
\hline $\mathrm{Bi}$ & A-7 & 42.7 & 16.5 & 59.2 & 57.1 \\
\hline $\mathrm{Ca}$ & $\mathrm{BCC}$ & 32.3 & 35.6 & 67.9 & \\
\hline $\mathrm{Cd}$ & HCP & 36.4 & 29.1 & 65.5 & \\
\hline $\mathrm{Co}$ & $\mathrm{FCC}$ & 143.6 & 160.0 & 303.6 & 263.9 \\
\hline $\mathrm{Cu}$ & $\mathrm{FCC}$ & 112.3 & 115.4 & 227.7 & 197.1 \\
\hline $\mathrm{Fe}$ & $\mathrm{BCC}$ & 123.8 & 140.5 & 264.3 & 226.9 \\
\hline $\mathrm{Ga}$ & $A-11$ & 31.0 & 21.7 & 52.7 & 61.3 \\
\hline $\mathrm{Ge}$ & $\mathrm{DC}$ & 163.8 & 38.2 & 202.0 & 188.2 \\
\hline $\mathrm{Hg}$ & $A-10$ & 12.2 & 9.6 & 21.8 & 27.5 \\
\hline $\mathrm{Li}$ & $\mathrm{BCC}$ & 18.6 & 17.5 & 36.1 & 30.0 \\
\hline $\mathrm{Mg}$ & HCP & 49.3 & 48.2 & 97.5 & \\
\hline $\mathrm{Mn}$ & $\mathrm{BCC}$ & 118.5 & 132.0 & 250.5 & 232.8 \\
\hline $\mathrm{Na}$ & $\mathrm{BCC}$ & 10.8 & 11.2 & 22.0 & 20.0 \\
\hline $\mathrm{Ni}$ & $\mathrm{FCC}$ & 156.6 & 168.6 & 325.2 & $\cdot 284.6$ \\
\hline $\mathrm{Pb}$ & FCC & 22.6 & 27.6 & 50.2 & 37.0 \\
\hline $\mathrm{Pd}$ & $\mathrm{FCC}$ & 126.2 & 125.9 & 252.1 & 231.9 \\
\hline $\mathrm{Pt}_{\mathrm{t}}$ & $\mathrm{FCC}$ & 162.3 & 149.7 & 312.0 & 267.1 \\
\hline $\mathrm{Sb}$ & A-7 & 84.3 & 31.2 & 115.5 & 105.6 \\
\hline $\mathrm{Si}$ & $\mathrm{DC}$ & 243.5 & 88.0 & 331.5 & \\
\hline Sn & A-5 & 33.1 & 20.5 & 53.6 & 59.3 \\
\hline
\end{tabular}
$\gamma_{\mathrm{CL}}$ (exp.) is taken from homogeneous nucleation. ${ }^{20)}$

transition zone adjacent to the interface, $k_{\mathrm{B}}$ the Boltzmann constant, $g(r)$ the pair distribution function of liquid and $b$ the cutoff distance (about $2 \mathrm{~nm}$ ) beyond which the function $g(r)$ indicates only an insignificant deviation from unity. Then, the contribution from the liquid near the interface in terms of the reduced liquid entropy is given by

$$
\gamma_{\mathrm{CL}}^{\mathrm{L}}=-T \Delta S_{\mathrm{CL}}
$$

Calculations by Eq. (5) with measured pair distribution functions have been made for 10 metals ${ }^{27-28)}$ and fair agreement between the calculated values and the experimental data by Turnbull and $\mathrm{Cech}^{20}$ ) was reported. This has been confirmed in more various metals ${ }^{29)}$ as shown in Table 3. In this work, a slightly modified equation was used for calculation of the quantity $\gamma_{\mathrm{CL}}^{\mathrm{s}}$ by introducing the concept of " $a$ random and high-index crystalline surface", ${ }^{29)}$ which enables us to estimate the values of $\gamma_{\mathrm{CL}}^{\mathrm{s}}$ for metals of not only a close packed 
structure but also any types of crystal structure. It may be added that Ewing's method gives the almost same order of magnitude with respect to two terms, $\gamma_{\mathrm{CL}}^{\mathrm{S}}$ and $\gamma_{C L}^{\mathbf{L}}$. Regarding this point, Spaepen ${ }^{30)}$ reported that the $\gamma_{\mathrm{CL}}^{\mathrm{S}}$ term increases by a factor of 2 in comparison with the case of the Ewing model for fcc and hep metals when a more strong crystallographic interaction is considered in the first layer of the liquid phase at the interface. However, no explicit comment on the superiority of each approach has not been given yet because no rigorous information of the liquid-solid interface in metals is available.

Ewing $^{26)}$ stressed by himself that the approach lacks rigor with respect to a few points;

(1) The density distribution of the liquid phase in the region of the interface is quite likely more localized than the bulk pair distribution function would indicate.

(2) The non-attracting hard sphere approximation in the derivation of spatial entropy is not well-recognized in liquid metals due to the rather soft repulsion and long-range oscillatory interaction.

(3) It seems somewhat artificial to associate the broken bond energy contribution only, because there are bulk liquid bonds as well as solid bonds broken at the liquid--solid interface.

Although these points should thoroughly be examined in due course, the results obtained are quite encouraging and then Ewing's approach represents a significant progress for discussing the liquid-solid interfacial energy of metals.

\subsection{Experimental Studies}

Any experimental study of atomic structures of the liquid-solid interface in metals has not been reported. In the present stage, several experimental studies which may give some structural information of the liquid-solid interface are the ones regarding with the surface melting phenomena (which is also called the surface roughening transition). The mechanism of melting is still a principal problem in condensed-matter physics and a thorough understanding of melting phenomena, at the microscopic level, has not been accomplished. Many theoretical works and computer simulations using the molecular dynamics have predicted that surfaces do, in fact, melt below the bulk equilibrium melting temperature $\left(T_{\mathrm{m}}\right)$. Molecular dynamic simulations of $\mathrm{Ni}(110)$ plane $^{31)}$ predicted that disordering of the surface region occurs first via anharmonic effects $(T<1450 \mathrm{~K})$, the generation of top layer vacancies accompanied by the formation of an adlayer at $T \sim 1450 \mathrm{~K}$, the gradual thickening of the defective surface region due to vacancy formation in deeper layers at $T>1450 \mathrm{~K}$, and the eventual formation of a quasi-liquid surface region (premelting) at $T \sim$ $1700 \mathrm{~K}$. Above $1700 \mathrm{~K}$, the rate of loss of long-range order in the crystal and the increase in diffusion catastrophically evolve. Incidentally, the melting temperature $T_{\mathrm{m}}$ of $\mathrm{Ni}$ is $1726 \mathrm{~K}$. Such a change of atomic structures on the surface during surface melting is estimated from an intensity profile of the X-ray reflectivity and from intensity variations of total external reflectance-diffraction peaks as a function of tempera- ture. The former method is the same as the one used in the studies of the liquid-vapor interface. As mentioned above, X-rays are strongly attenuated and their penetration is limited to a few $\mathrm{nm}$ deep in the total external reflection condition. Marra et al. ${ }^{32)}$ utilized this properties of X-rays together with the conventional Bragg scattering technique and proposed a new technique for the surface structural study by X-rays which is often called the GIXS (grazing incidence X-rays scattering). This is the method used in the latter experiment. The diffraction geometry in the GIXS is schematically shown in Fig. 5.

Diffuse scattering that originates in the abrupt change of density at a crystal surface has been reported. ${ }^{33,34}$ ) Such a discontinuity gives rise to rods of scattering in reciprocal space which are most intense close to the Bragg peaks and are well defined for sufficiently smooth surfaces. This diffuse intensity profile gives information on the topographic structure of the crystal surface. Figure 6 shows the observed diffuse intensity profile extending from (002) Bragg peak along the surface normal from the $\mathrm{Cu}(110)$ surface. $^{35)}$ By comparing this data with the theoretical intensity profiles, ${ }^{33,34)}$ the values of the root mean square roughness $\left\langle u^{2}\right\rangle^{1 / 2}$ determined are $0.146 \mathrm{~nm}$ at $543 \mathrm{~K}$ and $0.204 \mathrm{~nm}$ at $923 \mathrm{~K}$. A fair agreement between the excess width of the surface roughness at $923 \mathrm{~K}$ of $(0.142 \mathrm{~nm})^{2}\left[=(0.204 \mathrm{~nm})^{2}-(0.146 \mathrm{~nm})^{2}\right]$ and the height of a step on the (110) surface $(0.128 \mathrm{~nm})$ may be a strong

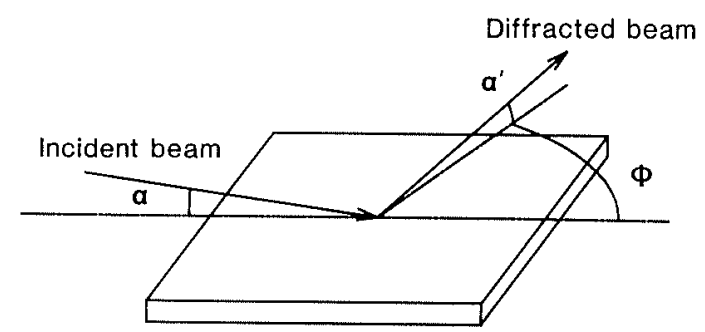

Fig. 5. Schematic diagram of the grazing incidence $X$-ray scattering (GIXS) geometry. The angles of $\alpha$ and $\alpha^{\prime}$ are angles of incidence and reflectance, respectively, and the angle $\Phi$ is a scattering angle.

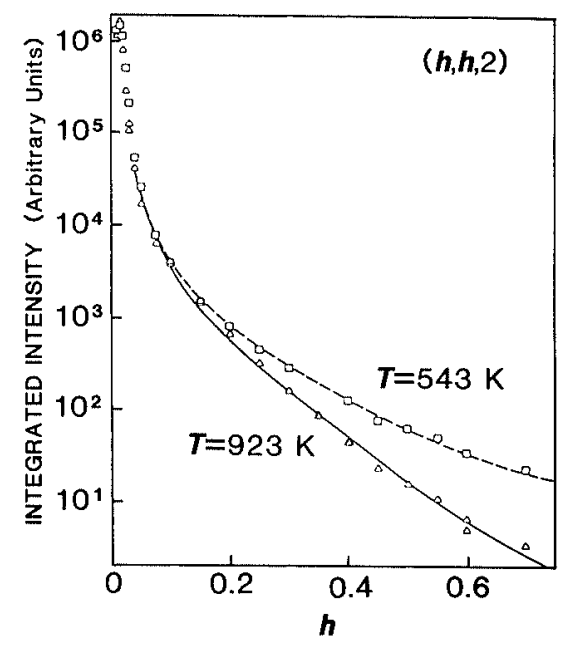

Fig. 6. Bragg reflectivity of $\mathrm{Cu}(110)$ at $T=543 \mathrm{~K}$ (squares) and $T=923 \mathrm{~K}$ (triangles) ${ }^{35)}$ The term $h$ is a coordinate in reciprocal space in units of $2 \pi / a$, where $a$ is the copper lattice constant. 
evidence of the surface roughening transition due to the step formation. ${ }^{35)}$ Other scans in Fig. 7 which correspond to scans transverse to the diffuse streak which extends between the (111) and the (111) Bragg peaks indicate that the surface roughening transition temperature is about $873 \mathrm{~K}^{\left.3{ }^{35}\right)}$ The similar observations have been reported on a different crystal surface. A liquid-like scattering profile in Fig. 8 was obtained from $\mathrm{Pb}$ (111) surface at $599 \mathrm{~K}^{36)}$ using the GIXS method. By analyzing this profile, Fuoss et al. ${ }^{36)}$ deduced the nearest neighboring correlations between $\mathrm{Pb}$ atoms on the (111) surface layer.

Certainly, these kinds of measurements to other materials and crystal faces are required for a complete understanding of the roughening of metal surfaces. The connection between macroscopic and microscopic interpretations of the interface is exciting and it is hoped that such experimental results will stimulate theoretical studies, elucidating the structure-properties relationship with relevant to the liquid-solid interface.

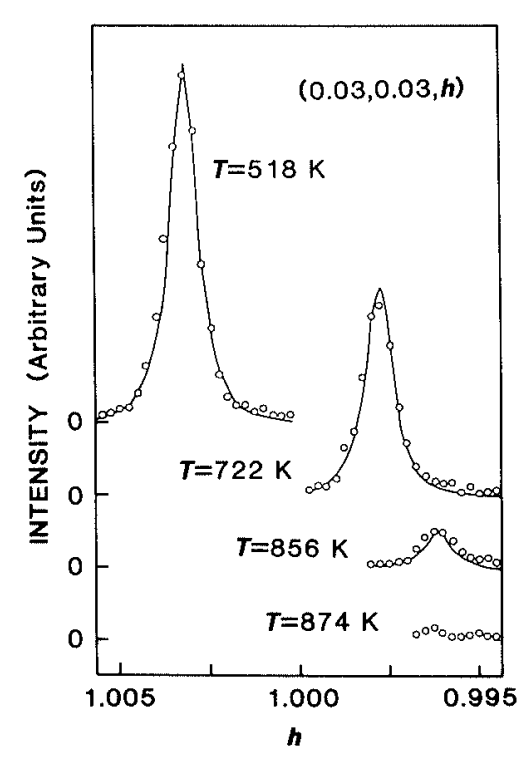

Fig. 7. Scans transverse to the Bragg reflectivity at (001). ${ }^{35)}$ The change in peak position is a result of thermal expansion. The term $h$ is the same meaning as that in Fig. 6 .

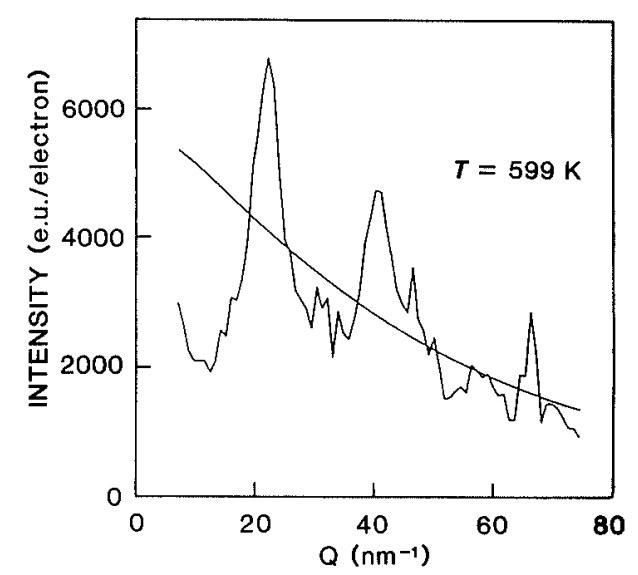

Fig. 8. Scattering intensity profile from the $\mathrm{Pb}$ (111) surface. ${ }^{36)}$

\section{Experiments with Fine Particles}

In this section, we are going to introduce a completely different experimental approach for studying the atomic structures of the surface. For fine particles with a crystalline size of $10 \mathrm{~nm}$, for example, about $15 \%$ of the total atoms are located on the surface. Thus, some physical properties of such fine particles are different from those of the bulk. For example, the melting point of fine particles is well known to drastically be reduced with decrease in their crystalline size, ${ }^{37)}$ which reminds us the surface melting phenomena discussed above. Harada and Oshima ${ }^{38)}$ estimated the Debye parameters from the X-ray Debye-Scherrer lines of the fine gold particles with a size of 6 to $23 \mathrm{~nm}$ at various temperatures. Using the least squares fitting of the Debye parameters as a function of temperature, the Debye characteristic temperatures $\Theta_{\mathrm{E}}$ for various sizes of fine particles were deduced on the basis of the Einstein approximation. The results are compared with the value of the bulk gold crystal in Table 4 . The results clearly indicate that the Debye characteristic temperature deviates from the bulk value with decrease in the particle size. Zhu et al. ${ }^{39)}$ also reported the structural information of the surface of fine $\mathrm{Fe}$ particles with a few $\mathrm{nm}$ size (they called nano-crystals) obtained by carefully measuring their X-ray scattering profile.

Figure 9 shows the scattering intensity profile of $\mathrm{Zn}$ ferrite crystalline particles with the average size of $4 \mathrm{~nm}^{40)}$ When the contributions from atoms on the surface of the particles with $0.2 \mathrm{~nm}$ deep and that from

Table 4. Comparison of Debye characteristic temperatures $\Theta_{\varepsilon}$ for fine gold particles with the value of the bulk crystal. ${ }^{38)}$

\begin{tabular}{cc}
\hline Particle size $(\mathrm{nm})$ & $\Theta_{\mathrm{E}}(\mathrm{K})$ \\
\hline 60 & 134 \\
115 & 145 \\
150 & 144 \\
235 & 161 \\
Bulk & 168 \\
\hline
\end{tabular}

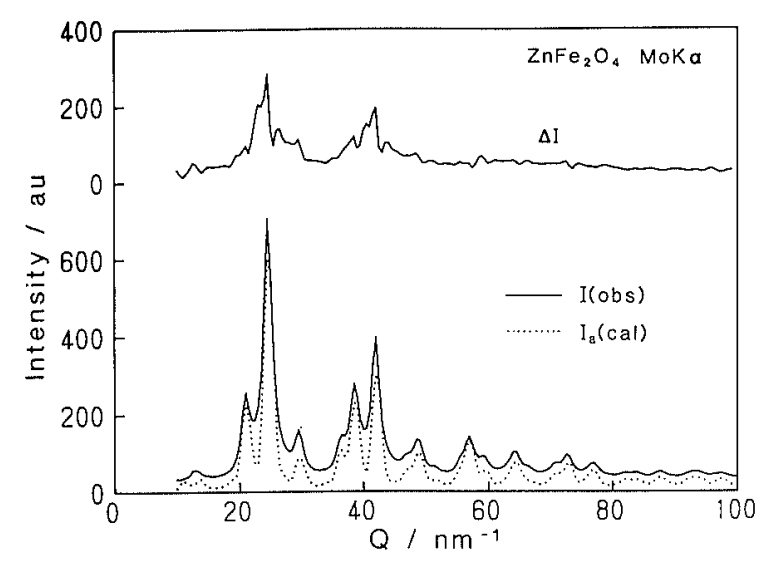

Fig. 9. Scattering intensity of $\mathrm{Zn}$ ferrite particles with a size of $4 \mathrm{~nm}$ (solid curve at the bottom), and the estimated contributions from the core (dotted curve at the bottom) and that from the surface with $0.2 \mathrm{~nm}$ deep. ${ }^{40}$ ) 


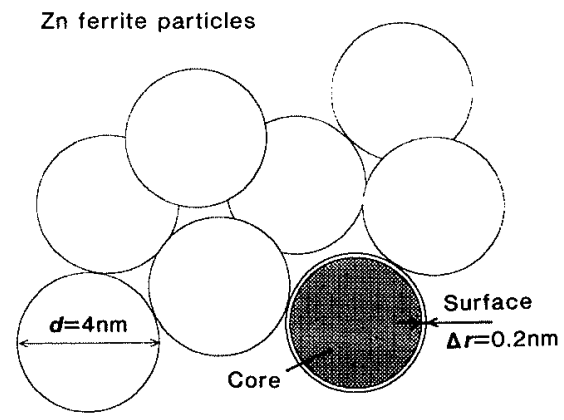

Fig. 10. Explanation of the core and surface of fine particles used for the computation of scattering intensity. ${ }^{40}$ )

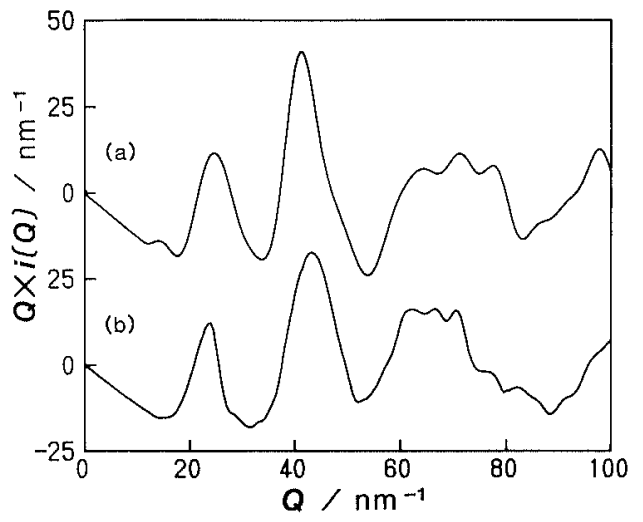

Fig. 11. Interference functions computed (a) from the intensity difference $\Delta I$ in Fig. 9 in $\mathrm{Zn}$ ferrite particles with a size of $4 \mathrm{~nm},{ }^{40)}$ and (b) from the scattering intensity in an amorphous $\mathrm{Zn}$ ferrite film grown on a silicate glass slide. ${ }^{411}$

the core (Fig. 10) are separately considered and the contribution to the scattering intensity in terms of the interference between these two regions is ignored for the simplification of the simulation, the calculated scattering intensity of the atoms in the core is given by the dotted curve in Fig. 9. The difference $\Delta I$ between this calculated intensity profile and the observed intensity profile (the solid curve in the bottom of Fig. 9) which is given in the top of the figure is considered as the scattering intensity from the surface of the particles. It is found in Fig. 11 that the interference function computed from this intensity difference is quite similar to the function of the amorphous $\mathrm{Zn}$ ferrite film. ${ }^{41)}$ This suggests that the atoms on the surface of the zinc ferrite particles have only a short-range ordering correlation similar to the one in the glass structure. These results fairly well indicate that the X-ray analyses of fine particles may give some valuable information of the atomic structure on the surface which is as good as the one determined from the smooth crystalline surface with the GIXS method.

\section{Concluding Remarks}

Some theoretical studies predicted that the density gradient at the liquid-vapor interface is relatively sharp with a couple of atomic layers thick, which is consistent with the experimental result by X-ray reflectivity measurements from the interface of liquid Cs. Although es- timating the liquid-solid interfacial energy made great progress in terms of Ewing's structural model for liquidsolid interface, no experimental result of the atomic structure of the interface has been reported. The only experimental result by $\mathrm{X}$-rays associated with atomic structures of the liquid-solid interface is the one regarding with the surface roughening transition in $\mathrm{Cu}$ and $\mathrm{Pb}$ single crystals, using the GIXS method. These studies suggest a liquid-like surface structure on the surface of metals well below their bulk equilibrium melting temperature. We believe that such experimental studies will provide us some hints about the atomic structure of the liquid-solid interface. As a completely different type of experimental approach, we mentioned the structural studies of fine particles with a size less than $10 \mathrm{~nm}$ using the conventional and anomalous X-ray scattering methods. Measurements in $\mathrm{Au}, \mathrm{Pd}$ and $\mathrm{Zn}$ ferrite particles, etc., have been considered one way to obtain some structural information about surface atoms

Unlike other techniques such as LEED (low energy electron diffraction), scattering intensities obtained from the interface using $X$-ray diffraction method are interpreted in a straightforward manner. In addition to the easy interpretation, the availability of intense synchrotron $\mathrm{X}$-ray radiation sources have made such measurements in structural studies of the interface practically feasible. Therefore, X-ray diffraction methods introduced above is becoming one of the most powerful techniques for structural studies of the interface.

\section{Acknowledgement}

A part of this research project on interfaces was sponsored by the Ministry of Education, Science and Culture of Japan for Grant-in-Aid for Scientific Research on Priority Areas under Grant No. 03243207 and for Grant-in-Aid for Encouragement of Young Scientists under Grant No. 03855140.

\section{REFERENCES}

I) J. G. Kirkwood and F. P. Buff: J. Chem. Phys., 17 (1949), 338.

2) A. Harashima: J. Phys. Soc. Jpn., 8 (1953), 343.

3) R. H. Fowler: Proc. R. Soc., A159 (1937), 229.

4) P. D. Shoemaker, G. W. Paul and L. E. Marce de Chazal: $J$. Chem. Phys., 52 (1970), 491.

5) M. V. Berry, R. F. Durrans and R. Evans: J. Phys. A (Gen. Phys.), 5 (1972), 166.

6) H. Eyring, D. Henderson, B. J. Stover and E. M. Eyring: Statistical Mechanics and Dynamics, Wiley, New York, (1964), Chap. 15.

7) P. A. Egelstaff and B. Widom: J. Chem. Phys., 53 (1970), 2667.

8) C. A. Croxton and R. P. Ferrier: Phys. Lett., 36A (1971), 183.

9) Y. Waseda and K. T. Jacob: to be submitted to High Temp. Mater. Process.

10) Y. Waseda and K. T. Jacob: Phys. Status Solidi (a), 68 (1981), K117.

11) H. L. Frisch and Z. W. Salsburg: Simple Dense Fluids, Academic Press, New York, (1968), Chap. 3.

12) B. C. Allen: Liquid Metals, ed. by S. Z. Beer, Marcel Dekker, New York, (1972), 161

13) A. H. Compton: Philos. Mag., 45 (1923), 1121.

14) L. G. Parratt: Phys. Rev., 95 (1954), 359.

15) D. Sluis and S. A. Rice: J. Chem. Phys., 79 (1983), 5658.

16) M. P. D'Evelyn and S. A. Rice: Phys. Rev. Lett., 47 (1981), 1844.

17) U. Mohanty and S. A. Rice: J. Chem. Phys., 79 (1983), 2482.

18) H. Mykura: Solid Surfaces and Interfaces, Dover, New York, 
(1966).

19) J. M. Blakely: Introduction to the properties of Crystal Surfaces, Pergamon, Oxford, (1973).

20) D. Turnbull and R. E. Cech: J. Appl. Phys., 21 (1950), 804.

21) K. A. Jackson and B. Chalmers: Can. J. Phys., 34 (1956), 473.

22) A. S. Skapski: Acta Metall., 4 (1956), 576

23) S. N. Zadumkin: Fiz. Met. Metalloved., 13 (1962), 24.

24) I. A. Kotze and D. Kuhlmann-Wisdorf: Appl. Phys. Lett., 9 (1966), 96.

25) D. Turnbull: Liquids, Structure Properties, Solid Interactions, ed. by T. J. Hughel, Elsevier, New York, (1965), 14.

26) R. H. Ewing: J. Cryst. Growth, 11 (1971), 22I.

27) R. H. Ewing: Philos. Mag., 25 (1972), 779.

28) O. Haida and T. Emi: Tetsu-to-Hagané, 63 (1977), 132.

29) Y. Waseda and W. A. Miller: Trans. Jpn. Inst. Met., 19 (1978), 546 .

30) F. Spaepen: Acta Metall., 238 (1975), 729.

31) E. T. Chen, R. N. Barnett and V. Landman: Phys. Rev. B, 41
(1990), 439

32) E. V. Marra, P. Eisenberger and A. Y. Cho; J. Appl. Phys., 50 (1979), 6927

33) S. R. Andrews and R. A. Cowley: J. Phys. C, 18 (1985), 6427.

34) D. A. Bruce: J. Phys. C, 14 (1981), 5195.

35) S. G. J. Mochrie: Phys. Rev. Lett., 59 (1987), 304.

36) P. H. Fuoss, L. J. Norton and S. Brennan: Phys. Rev. Lett., 60 (1988), 2046.

37) R. P. Berman and A. E. Curzon: Can. J. Phys., 52 (1974), 923.

38) J. Harada and K. Oshima: Surf. Sci., 106 (1981), 51.

39) X. Zhu, R. Birringer, U. Herr and H. Gleiter: Phys. Rev. B, 35 (1987), 9085 .

40) E. Matsubara, K. Okuda, Y. Waseda and T. Suzuki: Proc. the 108th Spring Annual Meeting, Jpn. Inst. Met., Sendai, (1991), 406.

41) E. Matsubara, K. Okuda, Y. Waseda, S. N. Okuno and K. Inomata: Proc. the 106th Annual Meeting, Jpn. Inst. Met., Sendai, (1990), 126. 